\title{
Coherence and population dynamics of chlorophyll excitations in FCP complex: Two-dimensional spectroscopy study
}

\author{
Vytautas Butkus, ${ }^{1,2}$ Andrius Gelzinis, ${ }^{1,2}$ Ramūnas Augulis, ${ }^{2}$ Andrew Gall, ${ }^{3}$ Claudia Büchel, ${ }^{4}$ Bruno Robert, ${ }^{3}$ \\ Donatas Zigmantas, ${ }^{5}$ Leonas Valkunas, ${ }^{1,2}$ and Darius Abramavicius ${ }^{1, a)}$ \\ ${ }^{1)}$ Department of Theoretical Physics, Faculty of Physics, Vilnius University, Sauletekio ave. 9-III, 10222 Vilnius, \\ Lithuania \\ ${ }^{2)}$ Center for Physical Sciences and Technology, Savanoriu ave. 231, 02300 Vilnius, \\ Lithuania \\ ${ }^{3)}$ Institut de Biologie et Technologies de Saclay, Bt 532, Commissariat l'Energie Atomique Saclay, \\ 91191 Gif sur Yvette, France \\ ${ }^{4)}$ Institut fr Molekulare Biowissenschaften, Universitt Frankfurt, Max-von-Laue-Strae 9, Frankfurt, \\ Germany \\ ${ }^{5)}$ Department of Chemical Physics, Lund University, P.O. Box 124, 22100 Lund, \\ Sweden
}

\begin{abstract}
The energy transfer processes and coherent phenomena in the fucoxanthin-chlorophyll protein complex, which is responsible for the light harvesting function in marine algae diatoms, were investigated at $77 \mathrm{~K}$ by using twodimensional electronic spectroscopy. Experiments performed on the femtosecond and picosecond timescales led to separation of spectral dynamics, witnessing evolutions of coherence and population states of the system in the spectral region of $\mathrm{Q}_{y}$ transitions of chlorophylls $a$ and $c$. Analysis of the coherence dynamics allowed us to identify chlorophyll (Chl) $a$ and fucoxanthin intramolecular vibrations dominating over the first few picoseconds. Closer inspection of the spectral region of the $\mathrm{Q}_{y}$ transition of Chl $c$ revealed previously not identified mutually non-interacting chlorophyll $c$ states participating in femtosecond or picosecond energy transfer to the Chl $a$ molecules. Consideration of separated coherent and incoherent dynamics allowed us to hypothesize the vibrations-assisted coherent energy transfer between $\mathrm{Chl} c$ and $\mathrm{Chl} a$ and the overall spatial arrangement of chlorophyll molecules.
\end{abstract}

Keywords: fucoxanthin-chlorophyll protein, multidimensional spectroscopy, 77K temperature, quantum coherence, intramolecular vibrations

\section{INTRODUCTION}

Diatoms are unicellular chromophyte algae inhabiting marine environment. They are the major players in photosynthesis, accounting for nearly a quarter of the global primary production 1 . Light-harvesting in diatoms is performed by their intrinsic membrane proteins, such as the fucoxanthin-chlorophyll protein (FCP) complex. FCP shares some structural and sequence homology with the light-harvesting complex II (LHCII) from the higher plants ${ }^{4}$, but contains more carotenoids per chlorophyll. Carotenoids fucoxanthins (Fx) strongly absorb blue-green photons with the longest propagation distances in water. Such adaptation allows diatoms to efficiently harvest sunlight in underwater conditions.

Information on the molecular structure of FCP is very limited; there is no crystallographic data of the complex. Several preliminary models of pigment organization in FCP, based on the assumption that the structure of FCP is similar to the LHCII, have been proposed ${ }^{5} \sqrt{8}$. These models, however, are currently limited for several reasons. First, only some pigment binding sites are conserved between FCP and LHCII. Second, the pigment composition and, thus, detailed functional roles

\footnotetext{
a) Electronic mail: darius.abramavicius@ff.vu.lt
}

in LHCII and FCP are markedly different, as the latter contain nearly as many fucoxanthin molecules as chlorophylls (Chl) - most probably $8 \mathrm{Fx}, 8 \mathrm{Chl} a$ and $2 \mathrm{Chl} c$ molecules ${ }^{79 \mid}$ while LHCII is composed of $8 \mathrm{Chl} a, 6$ Chl $b$ and 4 xanthophyll molecules $\mathbf{1 0}$.

Chl $c$ molecules enhance the absorption of blue photons in FCP, as well11. Ultrafast molecular excitation transfer from Chl c to Chl $a$ molecules was shown to occur at room temperature ${ }^{12}$. Nonetheless, the whole picture of the energy funnels in the FCP complex and the physical phenomena ensuring ultrafast energy transfer still needs to be detailed. Unfortunately, lack of structural information about FCP prevents detailed numerical studies. All the information about the inner workings of this complex comes solely from the experimental data and its analysis, which is often done in the light of the proposed model ${ }^{5+8}$. Therefore, it is important to obtain as much experimental data as possible to constrain and refine the current models.

The two dimensional (2D) electronic spectroscopy (ES) is a remarkable tool for the analysis of coherent and incoherent phenomena appearing in molecular excitation dynamics. The first and the most recognized results of 2D ES demonstrating complex behavior of the excitation evolution were delivered for the photosynthetic FennaMatthews-Olson (FMO) complex from purple bacteria ${ }^{13}$. Since then, 2D ES has been successfully applied for studies of a number of different systems; 2D ES es- 
sentially helped to establish or update existing kinetic schemes of light-harvesting antennae and reaction centers of plants $14-17$ and bacteria 20 . The $2 \mathrm{D}$ ES was also performed on the whole photosystem I complex demonstrating signatures of fast energy transfer ${ }^{21}$. In addition, it was utilized in studies of other molecular aggregates, for instance, polymers $\$ 22$, cylindrical (bi-tubular) J-aggregates 23 , quantum dots 24 etc.

Most of these findings were obtained from experiments and theoretical simulations performed using (or assuming) the knowledge of the structural organization of the pigment molecules (often with resolution of a few angstrms). Thus, these studies were valuable not only for widening a general understanding of the physical mechanisms within molecular systems, but also were convenient for testing and interpreting the outcomes of the new method of 2D ES. As the method itself has already been well approved and appreciated, it becomes useful to apply the developed tools of analysis of $2 \mathrm{D}$ spectra for more obscured molecular systems, for instance the FCP complex, and try to obtain more information about their structure/composition.

Recently, a few multidimensional spectroscopy studies of FCP reported valuable insight about various unknown characteristics of the complex. A novel method of twocolor two-dimensional spectroscopy allowed to map the the carotenoid-chlorophyll energy transfer pathways and resolved spectral heterogeneity of carotenoids in $\mathrm{FCP} 25$. It was found, that fucoxanthins in FCP are spectrally distinguishable and yet transfer energy to chlorophylls very efficiently. In our recent study 12 , dynamics of twodimensional spectra of Chl $a$ and Chl $c Q_{y}$ absorption bands at room temperature were analyzed and we reported ultrafast energy transfer between Chl $c$ and Chl $a$, with signatures of excitonic coupling. However, limited temporal and spectral resolution in these particular experiments did not provide the possibilities to fully resolve coherent dynamics or a more fine structure of the FCP spectrum.

Here we present results of the 2D ES of FCP obtained with higher spectral and temporal resolution than in our previous work ${ }^{12}$. To better discriminate the weak spectral features of FCP, experiments were carried out at $77 \mathrm{~K}$ instead of the room temperature. The 2D spectra were analyzed with both femtosecond and picosecond time steps, thus, providing us the possibility to separate coherent and incoherent processes. In this work, we show that there are at least two Chl $c$ molecules in FCP, which are clearly spectrally resolvable and participate in energy transfer on remarkably different timescales. We discuss that this might be related to certain spatial arrangement of Chl $a$ and $c$ molecules in the complex. Finally, the analysis of rich coherent dynamics during the first picosecond reveals signatures of $\mathrm{Chl} a$ and fucoxanthin intramolecular vibrations, the former of which might play a role in enhancing ultrafast $\mathrm{Chl} c$-to- $a$ energy transfer.

\section{MATERIALS AND METHODS}

Fucoxanthin-protein complex was extracted and purified from diatom Cyclotella meneghiniana. The procedure of purification and extraction is given in detail in Ref.26. The sample for the measurements was diluted with the glycero-buffer-detergent solution (10 mM Mes pH 6.5 buffer, $2 \mathrm{mM} \mathrm{KCl,} 0.03 \%$ (w/v) n-Dodecyl $\beta$ D-maltoside and $60 \%(\mathrm{v} / \mathrm{v})$ glycerol) to achieve optical density of about 0.3 at Chl $a \mathrm{Q}_{y}$ band in $0.5 \mathrm{~mm}$ cell.

The sample was placed in a $0.5 \mathrm{~mm}$ quartz cell and gradually cooled to $77 \mathrm{~K}$ in a liquid $\mathrm{N}_{2}$ bath cryostat (Oxford Instruments).

The setup for coherent two-dimensional electronic spectroscopy was based on the inherently phasestabilized design by Brixner et al.27. In more detail, $10 \mathrm{fs}, 100 \mathrm{~nm}$ full width at half maximum (FWHM) light pulses (3 nJ energy per pulse at the sample) centered at $615 \mathrm{~nm}$ were generated by home built noncollinear optical parametric amplifier were pumped by the Yb:KGW amplified laser system (Pharos, Light Conversion Ltd.) with repetition rate reduced to $7 \mathrm{kHz}$ by means of pulse picker. Diffractive-optics-based noncollinear four-wave mixing setup with phase-matched box geometry was used. The third-order signal was obtained by spectral interferometry with the help of a heterodyning local-oscillator pulse. Sensitivity and noise resistance was significantly enhanced by means of double modulation lock-in detection 28. Coherence time delay was scanned within -140-300 fs time range with 2 fs time step controlled by movable fused-silica wedges in beams $\vec{k}_{1}$ and $\vec{k}_{2}$. $2 \mathrm{D}$ spectra were obtained by processing the accumulated sequence by the Fourier transform method 29 . The absolute phase of the 2D spectrum was retrieved by matching its projection with the transient absorption spectrum 30 recorded with the same setup with only two beams (for pump and probe) in use. Real (absorptive) parts of the total (rephasing+nonrephasing) spectra were used in further analysis.

\section{RESULTS}

The absorption spectrum of FCP measured at $77 \mathrm{~K}$ is shown in Fig. 1 by the black solid line. The spectrum contains a well expressed band at $14895 \mathrm{~cm}^{-1}$ and a few weaker features around $15700 \mathrm{~cm}^{-1}$ and $17000 \mathrm{~cm}^{-1}$. The dominating band at $14895 \mathrm{~cm}^{-1}$ is known to represent the $\mathrm{Q}_{y}$ transition of the core pigment $\mathrm{Chl} a$. The feature at $15680 \mathrm{~cm}^{-1}$ was assigned to the $\mathrm{Q}_{y}$ transition of Chl $c$, while the origin of bands at around $16200 \mathrm{~cm}^{-1}$ and $17000 \mathrm{~cm}^{-1}$ were discussed to be reflecting the $\mathrm{Q}_{x}$ transitions of Chl $a$ and $\mathrm{Chl} c$, respectively ${ }^{12}$.

The spectrum of the laser pulse used in the $2 \mathrm{D} \mathrm{ES}$ experiment is shown by a gray shaded area in Fig. 1 . The spectrum was centered at around $16240 \mathrm{~cm}^{-1}$ with the FWHM of $\sim 2600 \mathrm{~cm}^{-1}$. The laser spectrum was intentionally shifted to the blue to enhance the signals 


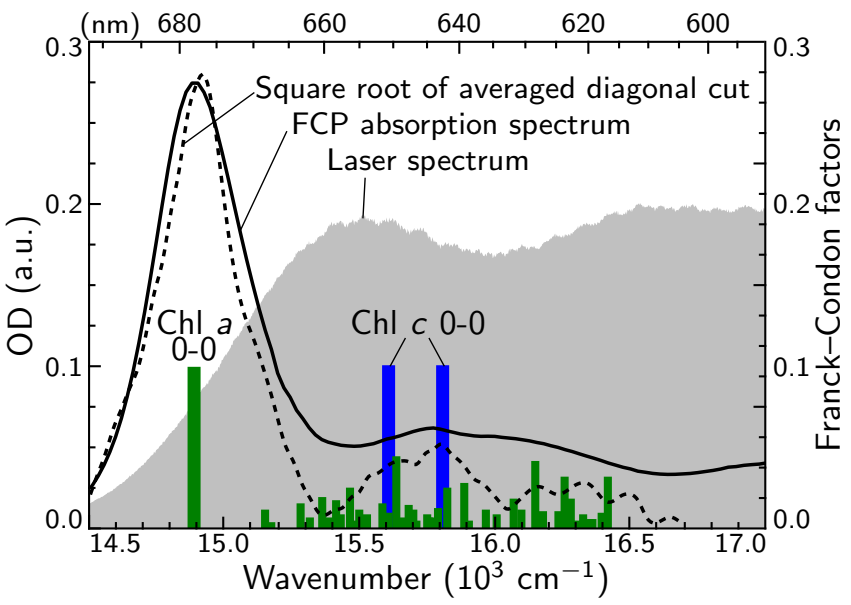

Figure 1. The absorption spectrum of FCP at $77 \mathrm{~K}$ (solid black line), square root of averaged (delay times from $t_{2}=$ $30 \mathrm{fs}$ to $800 \mathrm{fs}$ ) diagonal cut of the 2D spectrum (dashed black line), excitation laser spectrum of 2D ES experiment (filled gray area). Thicker vertical green and blue sticks indicate the energies of the $0-0 \mathrm{Q}_{y}$ transitions of Chl $a$ and Chl $c$, respectively. Vibrational states of Chl $a$, proposed by the hole burning measurements ${ }^{31}$ are shown by thinner green vertical sticks. Their heights indicate the Franck-Condon factors of the corresponding vibrations (values on the right axis).

form the weak absorption bands of FCP: while it only partly covered the main absorption band at $14895 \mathrm{~cm}^{-1}$, the other weak features were fully covered by the laser spectrum.

Two different 2D ES experiments were carried out. In the first one, the $2 \mathrm{D}$ spectra at delay times $t_{2}$ up to $800 \mathrm{fs}$ were taken at every $10 \mathrm{fs}$. Such a high temporal resolution is very useful for the analysis of the ultrafast excitation transfer dynamics as well as for studies of the quantum coherences and allowed us to resolve coherent beatings up to $1668 \mathrm{~cm}^{-1}$ in the frequency domain. The second set of data consisted of the 2D spectra measured at delay times $t_{2}$ up to $1 \mathrm{~ns}$ with different time steps: from 50 fs for the initial evolution to 100 ps for delay times longer than $100 \mathrm{ps}$. These measurements were used for the analysis of the incoherent long-time population dynamics in FCP.

A few 2D spectra at different delay times $\left(t_{2}=30 \mathrm{fs}\right.$, $330 \mathrm{fs}, 5 \mathrm{ps}$ and $30 \mathrm{ps}$ ) are depicted in Fig. 2 A. In the spectra, the main band corresponding to the Chl $a$ transition at $\omega_{3}=\omega_{1}=14926 \mathrm{~cm}^{-1}$ dominates. The fact that the band is not observed exactly at $14895 \mathrm{~cm}^{-1}$ as in the absorption spectrum, but rather blue-shifted by $31 \mathrm{~cm}^{-1}$ is caused by the excitation laser spectrum being slightly off-resonant with respect to the main FCP absorption band (see Fig. 1). The negative excited state absorption is well resolved above the diagonal in the 2D spectrum in the vicinity of the main positive absorption peak. The excited state absorption features in the 2DES could arise both due to excitonically coupled pigments (chlorophylls) and the corresponding double-excitation transitions ${ }^{32} \sqrt{34}$, as well as from the double-excitations of chlorophylls themselves 35 .

In the 2D spectra shown in Fig. 2, thinner contour lines, drawn at every $2 \%$ of the maximum highlight the fine structure of the diagonal and off-diagonal peaks (cross-peaks) away from the main Chl $a$ absorption band. The most pronounced features correspond to the crosspeaks lying around the same emission frequency $\omega_{3}$ as the Chl $a$ absorption band. These peaks might either signify the excitonic coupling between Chls $a$ and $c^{[12}$, or they might be caused by the $\mathrm{Chl} a$ vibrational modes.

In the region of the $\mathrm{Q}_{y}$ of the Chl $c$ transition frequency, i.e. at around $\omega_{3}=\omega_{1} \approx 15700 \mathrm{~cm}^{-1}$, two diagonal peaks are clearly resolved at excitation/emission frequencies $15610 \mathrm{~cm}^{-1}$ and $15810 \mathrm{~cm}^{-1}$. The positions of these peaks are indicated by two vertical bars in the absorption spectrum shown in Fig. 1, coinciding with the maxima of the diagonal cut of the averaged $2 \mathrm{D}$ spectrum (black dashed line in Fig. 11). The zoomed-in region of the $2 \mathrm{D}$ spectrum from $\omega_{3}=\omega_{1}=15300 \mathrm{~cm}^{-1}$ to $\omega_{3}=\omega_{1}=16100 \mathrm{~cm}^{-1}$ (shown by a red square in Fig. 2A) is depicted in Fig. $2 \mathrm{~B}$ at delay time $t_{2}=30 \mathrm{fs}$. No cross-peak associated with these two transitions is visible at any delay time disproving the presence of excitonic coupling between states at $15610 \mathrm{~cm}^{-1}$ and $15810 \mathrm{~cm}^{-1}$.

The 2D spectra contain rich dynamics both at short (femtosecond) and long (picosecond) delay times, as it was extracted from the separate measurements described above. Dynamics of a few selected peaks in the spectra, occurring during first $30 \mathrm{ps}$ are shown in Fig. $2 \mathrm{C}$. The main diagonal peak (indicated by "1-1") decays with timescales of $220 \pm 100 \mathrm{fs}$ and $5.4 \pm 0.6$ ps. The cross-peaks (indicated by " $2-1$ ", " $3-1$ " and " $4-1$ ") decay with the same longer rate of 5.4 ps as the "1-1" peak; the initial evolution is dominated by the high amplitude coherences (Fig. 2D) and, thus, shorter lifetimes could not be unambiguously evaluated. Diagonal peaks at $15610 \mathrm{~cm}^{-1}$ and $15810 \mathrm{~cm}^{-1}$ show completely different long-time dynamics. The upper peak decays much slower than the lower one, as it can be seen by the spectral traces in Fig. 2 $\mathrm{C}$. We could fit the dynamics of both peaks as single-exponential decays with timescales of $\tau_{15610}=320 \pm 70 \mathrm{fs}$ and $\tau_{15810}=3.9 \pm 1.6 \mathrm{ps}$, respectively. For longer delay times, additional exponential decays are present.

The short-time (femtosecond) evolution of the same points in the spectrum are shown in Fig. 2 $\mathrm{D}$ demonstrating the oscillating behavior of the the diagonal and off-diagonal peaks corresponding to the Chl $a$. Coherence beatings can be analyzed by performing the Fourier transform of the time traces of the peaks. A very convenient way to represent the overall complexity of oscillations in the $2 \mathrm{D}$ spectrum is through the Fourier maps 36 41]. They are constructed by performing the Fourier transform of the each data point in the 2D spectrum over the delay time $t_{2}$ after subtraction of slow exponential decay contributions. Then, the Fourier amplitude can be plotted as a two-dimensional map against 

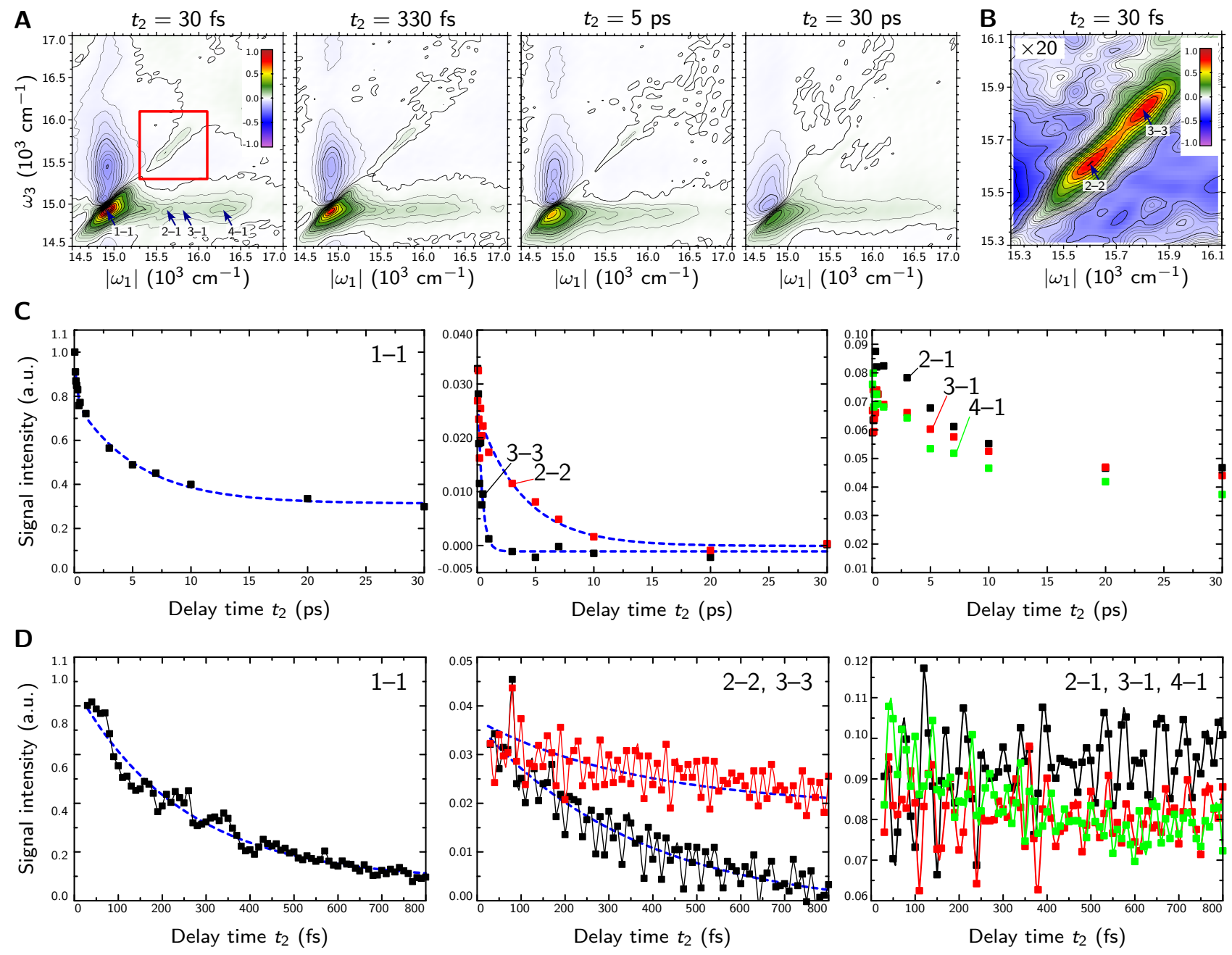

Figure 2. (A) 2D absorptive spectra at different delay times. All the spectra are normalized to the maximum of the spectrum at $t_{2}=30 \mathrm{fs}$ and drawn using the same scale. Thicker contour lines are drawn at every $10 \%$ of the maximum of the scale; thinner lines are drawn every $2 \%$, up to $\pm 20 \%$. (B) The two-peak structure in the $2 \mathrm{D}$ spectrum of FCP. Zoomed-in region, as shown in Fig. $2 \mathrm{~A}$ by the red square. Values in the spectrum are multiplied by 20 and the spectrum is drawn using the same scale as in (A). (C) Picosecond and (D) femtosecond time traces of a few selected peaks indicated by arrows in (A) and (B). Values obtained by averaging around the selected points within the circle of $100 \mathrm{~cm}^{-1}$ radius are presented. Multi-exponential fits of dynamics of a few peaks are shown by the blue dashed lines (see the text for the fitting parameters).

excitation and detection frequencies $\omega_{1}$ and $\omega_{3}$ for a fixed Fourier frequency $\omega_{2}$. Each Fourier map then can be integrated over $\omega_{1}$ and $\omega_{3}$ and the integral value represented as function of coherence frequency $\omega_{2}$. Such a dependency would show the averaged distribution of various oscillation frequencies.

We chose to represent the amplitude of observed oscillations by the value of the Fourier map norm, which is equal to the square root of the sum of squares of the data points of Fourier maps at frequency $\omega_{2}$. From the oscillation amplitude dependency shown in Fig. $3 \mathrm{~A}$, 5 sharp peaks at $\omega_{2}=255 \mathrm{~cm}^{-1}, 345 \mathrm{~cm}^{-1}, 745 \mathrm{~cm}^{-1} 1155 \mathrm{~cm}^{-1}$ and $1515 \mathrm{~cm}^{-1}$ can be readily pointed out. More rigorous analysis of the second derivative of the oscillation amplitude function reveals 24 more frequencies.

\section{DISCUSSION}

The 2D ES spectra obtained with femtosecond and picosecond time resolutions give a wealth of information regarding both evolution of population and coherent states of the molecular system under consideration. Special attention should be taken into consideration when analyzing the processes occurring on different timescales. Thus, before turning to the analysis of the femtosecond evolution of the measured spectra, let us discuss a few mechanisms that contribute to the spectral lineshape formation: pulse overlap, spectral diffusion, dynamic Stokes shift and coherence beatings. They can be important factors that might lead to ambiguities and errors when interpreting the time-dependencies of the $2 \mathrm{D}$ spectrum.

Pulse overlap effects dominate during the initial de- 
A

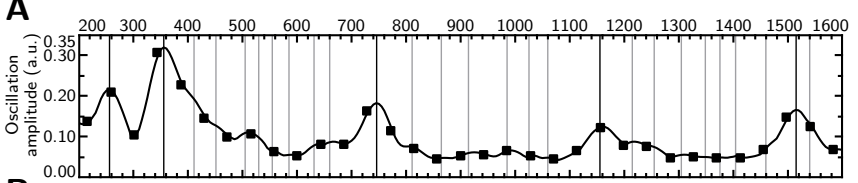

B

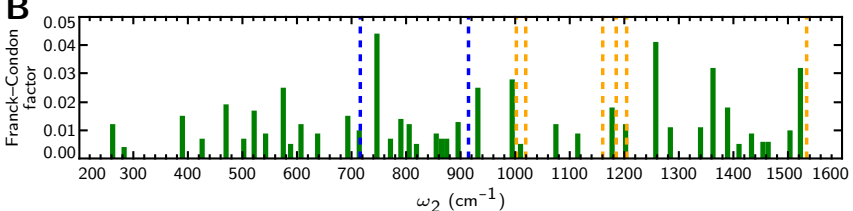

Figure 3. (A) Amplitude of oscillations (Fourier map norm) as a function of coherence frequency $\omega_{2}$. Dependency extracted from Fourier transforms of zero-padded and not-padded spectral time evolutions are shown by symbols and lines, respectively. Determined frequencies of oscillations are shown by the vertical black and gray lines. (B) Franck-Condon factors of vibrational intramolecular modes of Chl $a$ from the hole burning experiment ${ }^{31}$ (green sticks), proposed frequencies of electronic coherences (blue dashed lines) and vibrational Raman frequencies of fucoxanthin (orange dashed lines).

lay time evolution, comparable to the length of the laser pulses. That is, besides the double-coherence pathways, additional rephasing contributions appear in the phase matching direction when the $\vec{k}_{1}$ and $\vec{k}_{2}$ pulses do overlap ${ }^{42}$. Therefore, the initial evolution of the $2 \mathrm{D}$ spectrum (in our case first $30 \mathrm{fs}$ ) is dominated by the sharp decay of these additional pulse-overlap contributions and should be considered very carefully as they could be confused with some ultra-fast relaxation mechanisms.

Spectral diffusion can also make an evident influence on the $2 \mathrm{D}$ spectrum $\frac{43}{3}$. It accounts for the loss of correlation of the narrow-bandwidth excitation and spectrum is manifested by the increase of the anti-diagonal width of the peaks in the absorptive $2 \mathrm{D}$ spectrum with the delay time. The spectral diffusion affects both the rephasing and nonrephasing spectral contributions. For a simple two-level system the rate of the spectral diffusion can be related to the bath relaxation time and results in exponential decrease of the height of the main absorption peak in the 2D spectrum $\frac{44}{4}$. As such an exponential evolution overlaps with the other temporal lineshape formation mechanisms, it becomes troublesome not to overinterpret the spectral diffusion as a relaxation-type signal. Comparing the evolution of the peak height against its anti-diagonal width provides a possible way to evaluate the contribution of the spectral diffusion. However, sometimies this is not possible because of overlaps of closely-positioned coupled states and cross-peaks. The other method is to check the time evolution of the integral over some area of the spectrum in the vicinity of the peak under consideration in the absorptive spectrum. If the radius of the integrated area is larger than the homogeneous linewidth, the spectral diffusion is averaged out from such signal.

System states during the delay time $t_{2}$ experience relaxation processes through the vibronic pathways. Such a vibrational cooling is often referred to as the dynamic Stokes shift and expressing itself as the shifting of peaks down in the energy scale along the emission axis with $t_{2}$ in the $2 \mathrm{D}$ spectrum. As the ground state bleaching and excited state emission contributions can, in principle, experience the dynamic Stokes shift on different timescales, it can cause some overlaps of these positive and negative contributions, giving a complex time-dependence of both diagonal peaks and cross-peaks. Moreover, the timescale of the dynamic Stokes shift can be around a few hundreds of femtoseconds - therefore, without a careful analysis such evolution might once again be wrongly assigned to some ultrafast relaxation pathway.

Finally, the initial evolution of the $2 \mathrm{D}$ spectrum is seriously affected by the coherence beatings. Lifetimes of the electronic coherences can be as short as tens of femtoseconds and vibrational/vibronic coherences can result in oscillations for as long as a few picoseconds. Although static disorder tends to dephase electronic coherences very quickly, their contribution to the initial dynamics can still be substantial. For example, in spectra recorded with a low $t_{2}$ resolution, the coherent signal adding on top of the population evolution can be as significant as the population signal itself. Thus, coherence beatings can seriously impede interpretations of the population dynamics if the $2 \mathrm{D}$ spectrum is of low resolution in delay time $t_{2}$.

\section{A. Identification of coherences}

In the 2D ES spectra of FCP we would expect coherences of various origins and amplitudes to appear: ground state vibrational coherences of $\mathrm{Chl} a, \mathrm{Chl} c$ and Fx, excited state vibronic coherences of the same frequencies, mixed coherences of shifted or combinational frequencies $\frac{16 / 45}{1}$ and electronic coherences due to excitonic coupling of chlorophylls. Such distinction between the coherences of different origin is conveniently observed in the Fourier amplitude maps. In the rephasing spectrum, ground state coherences are manifested by strong beatings below and on the diagona ${ }^{38}$ while the corresponding vibronic coherences - symmetrically above the diagona 46-48. Mixed coherences, that represent superpositions of quantum mechanically mixed states, are observed as complex and asymmetric patterns of oscillating peaks in the Fourier maps $\$ 5 \mid 48$. Electronic coherences in the rephasing spectrum manifest themselves exclusively as oscillatory cross-peaks and no oscillations on the diagonal and vice versa for the nonrephasing spectrum 38 . However, oscillations in the nonrephasing contribution of the spectrum are more sensitive to static disorder of pigment energies and, thus, in the systems with large inhomogeneous broadening coherences from the rephasing contributions will dominate over those from the nonrephasing contribution.

Let us discuss about the origin of coherences in the spectra of FCP by comparing the obtained dependencies 
of the intensity of oscillations (Fig. 3A) with known frequencies corresponding to the intramolecular vibrational modes of Chl $a$. We refer to the hole burning experiments on single chlorophylls carried out in $1.6 \mathrm{~K}$ by Gillie et al! 31 (green sticks in Fig. 3B) and a recent study of $\mathrm{Du}$ et al. ${ }^{35}$, in which the frequencies were obtained from the time and frequency resolved pump-probe spectra at $297 \mathrm{~K}$ by the Fourier analysis, similar to the one employed here.

Frequencies, reported in these studies as well as obtained by our experiment, are summarized in Table I. The comparison gives almost perfect agreement not only for the obtained strongest coherences at around $\omega_{2}=$ $255 \mathrm{~cm}^{-1}, 345 \mathrm{~cm}^{-1}$, and $745 \mathrm{~cm}^{-1}$ (deviations smaller than $11 \mathrm{~cm}^{-1}$ ) but also for the weaker ones with the largest deviation of $20 \mathrm{~cm}^{-1}$ for the $\omega_{2}=1305 \mathrm{~cm}^{-1}$ vibrational mode. The Fourier amplitude maps at $\omega_{2}=$ $255 \mathrm{~cm}^{-1}, 345 \mathrm{~cm}^{-1}$, and $745 \mathrm{~cm}^{-1}$ are shown in Fig. 4 . All three of them clearly indicate the presence of vibrational and vibronic coherences as the patterns of the oscillatory features can be easily decomposed to 4 stimulated emission (SE) and 4 ground state bleaching (GSB) contributions of a simple two-level system with one vibrational state in the excited and ground states (see the scheme and the corresponding excitation pathways in Fig. 5A and $\mathrm{C}$ ). From the scheme it follows that weak features above the diagonal, separated by exactly $\omega_{2}$ value, indicate vibronic excited state coherences ( $\mathrm{SE}_{1}$ pathway).

The map at $\omega_{2}=745 \mathrm{~cm}^{-1}$ lacks amplitude of oscillations on the diagonal compared to the $\omega_{2}=345 \mathrm{~cm}^{-1}$ or $\omega_{2}=255 \mathrm{~cm}^{-1}$ maps due to the narrow bandwidth of the laser spectrum, suppressing some specific coherences. That is, since Chl a 0-0 transition is on the low-energy shoulder of the laser excitation spectrum, all excitation pathways containing lower energy transitions (indicated by the dashed arrows in Fig. 5 C) will be substantially suppressed. These particular pathways in Fig. $5 \mathrm{C}$ are shown in a shaded background.

For oscillations of higher frequencies, the mentioned contributions will be completely excluded. Therefore, the dependency of amplitude of oscillations in Fig. 3A should be considered carefully, as the peak heights there do not perfectly represent the amplitudes of all summed pathways, but are rather suppressed in middle and high frequencies.

The Fourier amplitude maps at $\omega_{2}=1155 \mathrm{~cm}^{-1}$ and $1515 \mathrm{~cm}^{-1}$ are shown in Fig. 4. They both contain broad features in the region of excitation frequency $\omega_{1}=16500-17000 \mathrm{~cm}^{-1}$ and emission frequency $\omega_{3} \approx$ $\omega_{1}-1155 \mathrm{~cm}^{-1}$ and $\omega_{3} \approx \omega_{1}-1515 \mathrm{~cm}^{-1}$, respectively. With our laser spectrum we could in principle excite the $\mathrm{S}_{2}$ states of some of the "red" fucoxanthins and, possibly, get signatures of carotenoid ground state coherences (from the $\mathrm{GSB}_{3}^{\mathrm{Fx}}$ pathway shown in Fig. 5D). These contributions would appear as oscillations at the emission frequency, shifted down from the diagonal by the frequency of the vibrational mode. Therefore, we assign these features observed at $\omega_{2}=1155 \mathrm{~cm}^{-1}$ and

\begin{tabular}{|c|c|c|c|c|c|c|c|}
\hline \multirow{2}{*}{$\overline{\mathrm{FCP}}$} & \multicolumn{2}{|c|}{ Chl $a$} & \multirow{2}{*}{$\begin{array}{l}\text { Fx } \\
\text { RR }\end{array}$} & \multirow{2}{*}{ FCP } & \multicolumn{2}{|c|}{ Chl $a$} & \multirow{2}{*}{$\begin{array}{l}\text { Fx } \\
\text { RR }\end{array}$} \\
\hline & $\overline{\mathrm{HB}}$ & $\mathrm{PP}$ & & & HB & $\overline{\mathrm{PP}}$ & \\
\hline 135 & - & - & - & - & 864 & - & - \\
\hline 190 & - & 214 & - & - & 874 & - & - \\
\hline 255 & 262 & 259 & - & - & 896 & - & - \\
\hline - & 283 & 300 & - & 915 & 932 & 915 & - \\
\hline 345 & - & 346 & - & 985 & 994 & 982 & 1002 \\
\hline- & 390 & - & - & 1025 & 1009 & 1043 & 1019 \\
\hline 410 & 425 & 407 & - & 1060 & 1075 & 1084 & - \\
\hline 450 & 469 & - & - & - & 1114 & 1124 & - \\
\hline 505 & 501 & - & - & 1155 & - & - & 1160 \\
\hline 530 & 521 & 519 & - & - & 1178 & 1175 & 1185 \\
\hline 555 & 541 & 565 & - & 1215 & 1203 & - & 1205 \\
\hline- & 574 & - & - & 1255 & 1259 & 1252 & - \\
\hline 585 & 588 & - & - & 1305 & 1285 & - & - \\
\hline- & 607 & - & - & 1350 & 1340 & 1353 & - \\
\hline 630 & 638 & 621 & - & 1375 & 1364 & - & - \\
\hline 660 & - & 667 & - & - & 1390 & - & - \\
\hline- & 692 & - & - & 1405 & 1411 & 1415 & - \\
\hline- & 714 & - & - & - & 1433 & - & - \\
\hline 745 & 746 & 744 & - & - & 1455 & - & - \\
\hline- & 771 & - & - & 1460 & 1465 & 1460 & - \\
\hline- & 791 & - & - & - & 1504 & - & - \\
\hline 810 & 805 & 799 & - & 1515 & 1524 & 1516 & - \\
\hline- & 819 & - & - & 1540 & - & - & 1536 \\
\hline 865 & 855 & - & - & & & & \\
\hline
\end{tabular}

Table I. Summary of coherent oscillations $\left(\mathrm{cm}^{-1}\right)$ in the measured FCP 2D spectrum (first column). Vibrational frequencies of chlorophyll $a$ obtained from the hole burning ${ }^{31}$ (HB, second column) and pump-probe ${ }^{35}$ (PP, third row) experiments. Vibrational frequencies of fucoxanthin from the resonant Raman (RR) study ${ }^{7}$ are shown in the fourth row. Numbers in bold denote the five most intensive oscillations in $2 \mathrm{D}$ and pump-probe experiments, two most intensive Raman modes of fucoxanthin and frequencies from the hole-burning experiment, having Franck-Condon factors higher than 0.02.

$\omega_{2}=1515 \mathrm{~cm}^{-1}$ to the ground state vibrational modes of $\mathrm{Fx}$ at $1160 \mathrm{~cm}^{-1}$ and $1536 \mathrm{~cm}^{-1}$, respectively ${ }^{7}$.

Apart from the Fx vibrational coherence, the Fourier amplitude map at $\omega_{2}=1515 \mathrm{~cm}^{-1}$ shows oscillations at $\omega_{3}=14935 \mathrm{~cm}^{-1}$ and $\omega_{1}=16550 \mathrm{~cm}^{-1}$ and a corresponding diagonal-symmetric feature. They can be assigned to the Chl $a$ vibrational and vibronic coherences, respectively. Thus, the full pattern of features in the map at $\omega_{2}=1515 \mathrm{~cm}^{-1}$ can be explained by the scheme shown in Fig. 5B: the two diagonal-symmetric narrow features come from the excited state vibronic and ground state vibrational coherences of $\mathrm{Chl} a$ and the broad feature below the diagonal is due to vibrational ground state coherences of Fx. The other weak features at the emission frequency $\omega_{3} \approx 16000 \mathrm{~cm}^{-1}$ cannot be explained by any sort of excitation pathways; we assign them to experimental artifacts coming from the nonrephasing contribution (not shown).

The Fourier map at $\omega_{2}=1155 \mathrm{~cm}^{-1}$ clearly lacks similar features stemming from $\mathrm{Chl} a$ vibrational coherences and there is no vibrational mode of similar frequency suggested by the hole-burning or pump-probe experiments 

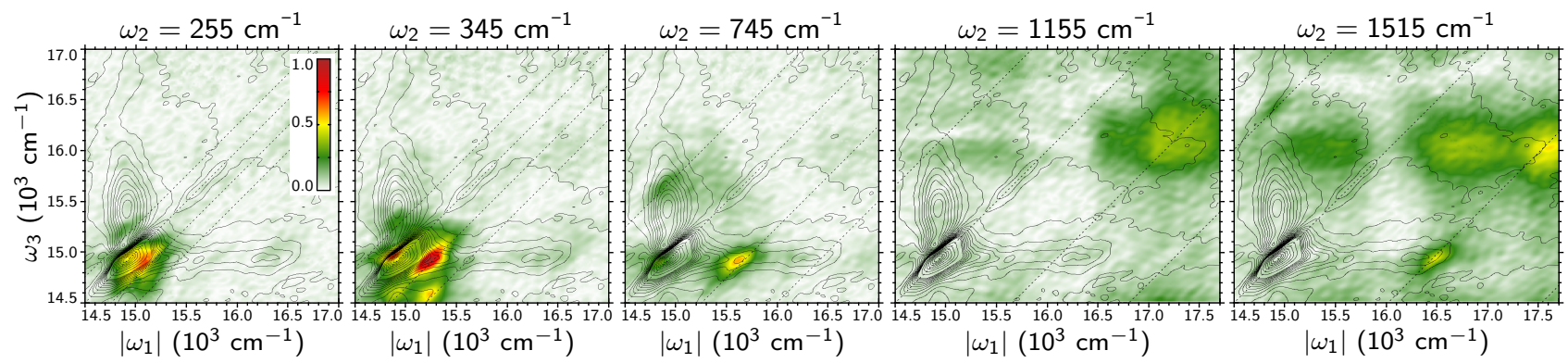

Figure 4. Fourier amplitude maps at coherence frequencies $\omega_{2}=255 \mathrm{~cm}^{-1}, 345 \mathrm{~cm}^{-1}, 745 \mathrm{~cm}^{-1}, 1155 \mathrm{~cm}^{-1}$ and $1515 \mathrm{~cm}^{-1}$. Fourier amplitude is shown by a color scale, normalized to the maximum of the map at $\omega_{2}=345 \mathrm{~cm}^{-1}$. Black contour lines show the $2 \mathrm{D}$ spectrum at $t_{2}=30 \mathrm{fs}$. Diagonal and parallel to the diagonal dashed lines are shifted by the corresponding value of $\omega_{2}$. Notice that excitation axex of maps at $\omega_{2}=1155 \mathrm{~cm}^{-1}$ and $1515 \mathrm{~cm}^{-1}$ are extended compared to the other maps.

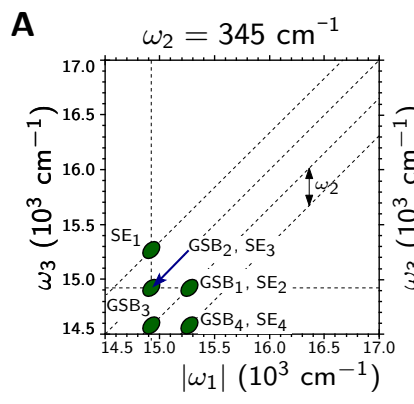

C
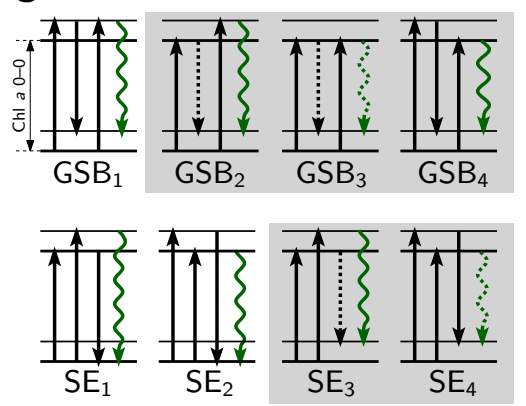

tional mechanisms, responsible for some vibrational Chl $a$ frequencies not being detected in FCP, e.g. vibronic coupling between $\mathrm{Chl} a$ in FCP, adding more complexity to spectra, similar to the enhancement of the ground state coherence proposed by Chenu et a $\sqrt{50}$, or the anharmonicity of the electron-vibrational coupling recently proposed for the LH2 antenat ${ }^{51}$. Obviously, the situation calls for more delaited study of vibrational/vibronic coherences of chlorophylls in coupled pigment-protein complexes.

After performing analysis of the Fourier maps of the other coherences, we were not able to extract any oscillatory features that could be assigned to the vibrational/vibronic coherences of $\mathrm{Chl} c$. This is probably due to too weak signal. Indeed, the $\mathrm{Chl} c$ absorption signal is at least 5 times weaker than the main absorption band of Chl $a$. Thus, the signal of coherent oscillations might become too weak to be distinguished from the experimental noise. A close inspection of oscillations around the proposed $\mathrm{Q}_{y}$ transition band of $\mathrm{Chl} c\left(\sim 15600 \mathrm{~cm}^{-1}\right)$ revealed only very weak signatures of vibrational coherences of Chl $a$ and Fx. The former might be coming from the nonrephasing vibrational contribution and the latter - from the overlap of a wide peak of the fucoxanthin ground state vibrational coherence.

Obviously, the strongest high-frequency electronic coherence in the spectra of FCP would be the coherence of $\mathrm{Q}_{y}$ transitions of excitonically coupled $\mathrm{Chl} c$ and Chl $a$. From the position of the corresponding peaks in the absorption spectrum, the frequency of such a coherence should be around $715 \mathrm{~cm}^{-1}$ or $915 \mathrm{~cm}^{-1}$. By comparing the peak heights and assuming that the Franck-Condon factors of vibrational transitions are as small as 0.05 , it could easily be deduced that the amplitude of electronic coherence should be higher than the vibrational coherence of Chl c. But the dephasing rate of the electronic coherences is known to be much shorter than the vibrational ones ${ }^{48}$. This would prevent the electronic coherence to be captured by the Fourier maps, except of a few cases. One of them is the inhomogeneous distribution induced picosecond electronic coherence lifetime, as it was recently shown by Dong et al. ${ }^{52}$. In the inhomogeneously broadened spectrum the sub-ensemble of molecules, hav-

considered here ${ }^{31 / 35}$. However, vibrational Chl $a$ mode of $1145 \mathrm{~cm}^{-1}$ has been reported in the fluorescence narrowing and resonant Raman study by Telfer et al 49 . It is possible that we were not able to resolve signatures of this particular vibrational coherence because of the suppression of high frequency coherences by laser spectrum as discussed above. Alternatively, there could be addi- 
ing similar energies provides long-lived coherent signal in the two-color photon echo experiment. Such mechanism could also be significant in the $2 \mathrm{D}$ electronic spectroscopy, however, it requires the absorption spectra of Chl $c$ and Chl $a$ to overlap significantly.

Another mechanism allowing for long-lived electronic coherences is substantial electronic-vibrational mixing in the excited state, resulting in a vibrational coherence lifetime borrowing mechanism 47 . For this effect to be noticeable, the excitonic energy gap between the excitonic states should closely match some strong discrete vibrational mode. In our case, the proposed excitonic energy gap is close to a very strong vibrational modes of $745 \mathrm{~cm}^{-1}$ or $915 \mathrm{~cm}^{-1}$ and in the Fourier amplitude maps the ground state vibrational contribution dominates. Therefore, if there are observable signatures of electronic coherence beatings, they are hindered by strong signals of vibrational coherences.

\section{B. Ultrafast $\mathrm{Chl} c-\mathrm{Chl} a$ transfer}

In our previous study of the $2 \mathrm{D}$ spectra of the $\mathrm{FCP}$ complex at room temperature, the ultrafast energy transfer (occurring on timescale of $60 \mathrm{fs}$ ) from excitonically coupled Chl $c$ to Chl $a$ was proposed 12 . It was obtained by tracing the time dynamics of the spectral feature at $\omega_{3}=\omega_{1}=15680 \mathrm{~cm}^{-1}$, assigned to the $Q_{y}$ transition of $\mathrm{Chl} c$. Here we report that there are two peaks at $15610 \mathrm{~cm}^{-1}$ and $15810 \mathrm{~cm}^{-1}$ instead of one. This doublet was not resolved in the experiment at the room temperature. Moreover, we found significant differences in their decay dynamics. Considering times up to $30 \mathrm{ps,}$ the lower peak decays with the timescale of 320 femtoseconds, while the the shortest temporal dynamics of the upper peak is $3.9 \mathrm{ps}$. The timescales were obtained from temporal traces, taken by averaging over the area of a circle of $100 \mathrm{~cm}^{-1}$ radius in spectra and omitting the first $30 \mathrm{fs}$, thus limiting amount spectral diffusion and pulse overlap contributions appearing in peak dynamics. We can thus conclude that the obtained two different $\mathrm{Chl} c$ states directly transfer energy to $\mathrm{Chl} a$ on different timescales. Notice that energy transfer via intermediate states (presumably from fucoxanthins) is not consistent with fluorescence-excitation spectrum of $\mathrm{FCP}$, which implies $100 \%$ efficiency of $\mathrm{Chl} c$ to $\mathrm{Chl} a$ energy transfer, and the fact that significant portions of excitation from Fx $\mathrm{S}_{1} / \mathrm{ICT}$ (internal charge transfer) state, which could be suggested to be a bridge between Chl $c$ and $\mathrm{Chl} a$, are lost due to the decay to the ground state ${ }^{25}$.

Recent studies suggest that there are two Chl $c$ molecules in the complex ${ }^{7}$. Also, some of them can be of different types (i.e. Chl $c_{1}, c_{2}$ or $c_{3}$ ) that have slightly different absorption spectra ${ }^{53}$. Even if the discussed spectral features are caused by the chlorophylls of different types (thus, causing signals in different frequencies), the temporal dynamics of the corresponding peaks indicate that their interaction with the rest of the system or a surrounding environment is different. The simplest picture is that one $\mathrm{Chl} c$ is situated in the periphery of the FCP complex and is weakly coupled to the rest of Chl $a$, thus, the excitation transfer to the core pigments is slow and we observe a picosecond decay of the peak at $15810 \mathrm{~cm}^{-1}$. At the same time, the protein environment causes it to have higher energy. Another Chl $c$, absorbing at $15610 \mathrm{~cm}^{-1}$, must be coupled to Chl $a$ stronger for faster excitation transfer. Since the cross-peak between these two transitions as well as the relevant excited state absorption contribution is absent, chlorophylls $c$ in FCP are not coupled. This implies that they probably are spatially well separated.

However, the ultrafast energy transfer from the lower energy Chl $c$ state still remains an issue to be explained. The strength of coupling between $\mathrm{Chl} c$ and $\mathrm{Chl} a$ is not known, although it may be estimated from LHCII, which shares considerable sequence homology ${ }^{4}$. Couplings between chlorophylls in the LHCII complex were calculated to be less than $100 \mathrm{~cm}^{-154}$. Considering this and the fact that the transition dipole moment of the $\mathrm{Q}_{y}$ band is smaller for Chl $c$ than for $\mathrm{Chl} a$, one can be certain that coupling between $\mathrm{Chl} c$ and Chl $a$ in FCP should be even weaker. This is also supported by the absence of clear excitonic cross-peaks between $\mathrm{Chl} a$ and $\mathrm{Chl} c$ states in our 2D spectra.

The Förster energy transfer could apply in this range of parameters. However, it leads to slower picosecond transfer rates. The $320 \mathrm{fs}$ transfer timescale observed in 2D ES can be understood by including two ingredients in the consideration. First, the lower energy $\mathrm{Chl} c$ can be coupled to several Chl $a$ molecules, thus, it transfers energy to a few $\mathrm{Chl} a$ molecules at once. The rates effectively sum-up and lead to the fast decay of the $\mathrm{Chl} c$ population. Second, vibrations-assisted energy transfer enhancement might be significant for the Chl $c$-Chl $a$ energy transfer. The energy gap of $715 \mathrm{~cm}^{-1}$ is close to the strongest vibrational Chl $a$ mode of $745 \mathrm{~cm}^{-1}$, thus allowing for electronic-vibrational resonance 55 57. Recently, specific vibrational modes were suggested to speed up the initial charge transfer step in the photosystem II (PS II) reaction center 16 .17]. Finally, this does not contradict our previous suggestion that $\mathrm{Chl} c$ is coherently coupled to $\mathrm{Chl} a^{12}$, however, the coherent mechanism may be far more complicated and calls for future studies.

We propose a schematic spatial arrangement of Chl $a$ and $\mathrm{Chl} c$ molecules (shown in Fig. 6). This functional scheme is comparable to the CP29 photosynthetic antenna of photosystem II $58 / 59$. The structure of the CP29 complex is similar to the light harvesting complex LHCII, which shares the sequence homology with FCP. In CP29 there are eight Chl $a$ and four Chl $b$ (the absorption maximum of Chl $b$ is around $15674 \mathrm{~cm}^{-1}$, similarly as to Chl c) and one more chlorophyll binding site can be occupied by either type of chlorophyl] $\left[\frac{59}{}\right.$, although previously it was assumed that there are $8 \mathrm{Chl} a$ and $2 \mathrm{Chl} b$ molecules 58 . However, in both models one chlorophyll $b$ molecule is well spatially separated from the other which is strongly 


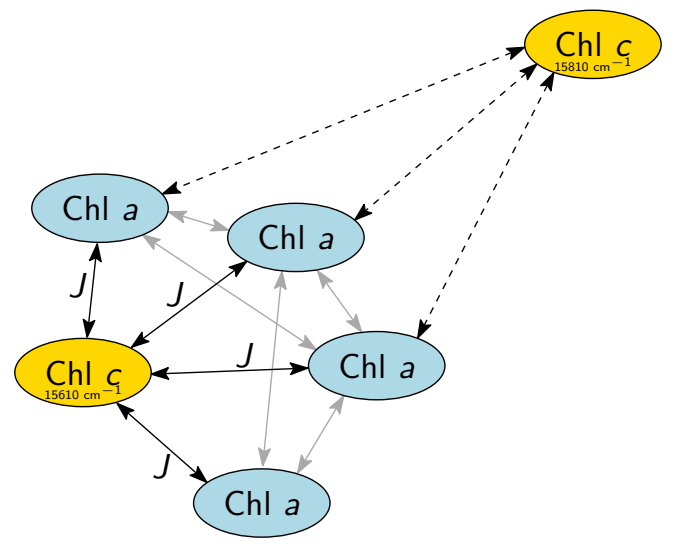

Figure 6. Proposed functional scheme of spatial arrangement of chlorophyll $c$ and $a$ molecules in the FCP complex. Couplings between pigments are shown by solid lines. Dashed lines indicate weaker coupling between spatially separated Chl $c$ absorbing at $15810 \mathrm{~cm}^{-1}$. Chl $a$ molecules shown here do not represent their actual number in FCP.

coupled to the rest of chlorophylls $a$ of the complex. Consequentially, two absorption bands at $15650 \mathrm{~cm}^{-1}$ and $15385 \mathrm{~cm}^{-1}$ are observed in the absorption and in the pump-probe spectrum have completely different temporal dynamics: the energy transfer from $\mathrm{Chl} b$ at higher energy is transferred to Chl $a$ with the rate of $350 \mathrm{fs}$, while transfer from the lower energy $\mathrm{Chl} b$ band occurs in $2.2 \mathrm{ps} 558$. This implies that having two accessory chlorophylls that are in different spatial situations might be a property shared by a number of light-harvesting complexes, thus probably it has a functional significance.

In a recent study of FCP based on resonance Raman technique ${ }^{7}$, signatures of two distinct $\mathrm{Chl} c$ molecules were observed. In that study a possible pigment organization scheme in FCP was discussed, based on the pigment binding sites of the LHCII from plants10. It was suggested that one of the Chl $c$ should be bound at either the site of a614 (which is preserved in FCP) or a613 (not preserved in $\mathrm{FCP}$ ), while the other Chl $c$ should be bound at the site of b609 (preserved) or a604 (not preserved). For the faster transferring Chl $c$ the site of b609 would be a logical choice, as it is in the vicinity of several pigments. Meanwhile, the higher energy $\left(15810 \mathrm{~cm}^{-1}\right) \mathrm{Chl} c$ could indeed be situated at the site of a614, as that site is relatively isolated and would be in accord with slower energy transfer from this pigment. Since this pigment is situated at the periphery of the complex, it might be responsible for the inter-complex $\mathrm{Chl} c$ to $\mathrm{Chl} a$ transfer in the FCP-PSII supercomplexes ${ }^{60}$.

\section{v. CONCLUSIONS}

In this work we studied low-temperature and highresolution experimental two-dimensional spectra of FCP complex. Short time (femtosecond) dynamics of the spec- tra show complex oscillation patterns. They are dominated by vibrational and vibronic coherences of $\mathrm{Chl} a$ and fucoxanthin; electronic coherences could not be identified due to their short lifetime and being hindered under high-amplitude oscillations originating from $\mathrm{Chl} a$. Most notably, we have resolved two distinct states, that can be assigned to chlorophylls $c$. These states were not found in the previous studies of FCP and their energies were not known. The two Chl $c$ molecules transfer energy to Chl $a$ with rates that differ by about an order of magnitude. We suggest that the lower energy $\mathrm{Chl} c$ molecule is situated in the core of the complex and is coupled to an aggregated pool of $\mathrm{Chl} a$ molecules and the Chl $c$-to$a$ energy transfer might be vibronically-enhanced. The higher energy $\mathrm{Chl} c$ should be in the periphery of the complex to transfer energy on the picosecond timescale.

\section{ACKNOWLEDGMENTS}

Authors would like to thank Kerstin Pieper for preparing the FCP samples and Egidijus Songaila for his work during the sample preparation procedure for the 2D ES experiment.

The research was partially funded by the European Social Fund under the Global Grant measure. Authors acknowledge support by LASERLAB-EUROPE project (grant agreement $n^{\circ} 228334$, EC's Seventh Framework Programme). Work in Lund was also supported by the Swedish Research Council and Knut and Alice Wallenberg Foundation. V. B. acknowledges support by project "Promotion of Student Scientific Activities" (VP1-3.1-MM-01-V-02-003) from the Research Council of Lithuania. C. B. acknowledges funding by the Deutsche Forschungsgemeinschaft (Bu 812/4-1, 5-1). C. B., A. Gall and B. R. acknowledges funding from the EU (HARVEST Marie Curie Research Training Network (PITN-GA-2009-238017). B. R. acknowledges support from the European Research Council (ERC) through an Advanced Grant, contract no. ERC-2010-AdG PHOTPROT.

\section{REFERENCES}

${ }^{1}$ P. G. Falkowski, R. T. Barber, and V. Smetacek, Science 281, 200 (1998).

${ }^{2}$ C. B. Field, M. J. Behrenfeld, J. T. Randerson, and P. Falkowski, Science 281, 237 (1998).

${ }^{3}$ D. G. Mann, Phycologia 38, 437 (1999).

${ }^{4}$ M. Eppard and E. Rhiel, Mol. Gen. Genet. 260, 335 (1998).

${ }^{5}$ E. Papagiannakis, I. H. M. van Stokkum, H. Fey, C. Büchel, and R. van Grondelle, Photosynth. Res. 86, 241 (2005).

${ }^{6}$ C. Wilhelm, C. Büchel, J. Fisahn, R. Goss, T. Jakob, J. LaRoche, J. Lavaud, M. Lohr, U. Riebesell, K. Stehfest, K. Valentin, and P. G. Kroth, Protist 157, 91 (2006).

${ }^{7}$ L. Premvardhan, B. Robert, A. Beer, and C. Büchel, Biochim. Biophys. Acta 1797, 1647 (2010).

${ }^{8}$ N. Gildenhoff, J. Herz, K. Gundermann, C. Büchel, and J. Wachtveitl, Chem. Phys. 373, 104 (2010). 
${ }^{9}$ L. Premvardhan, L. Bordes, A. Beer, C. Büchel, and B. Robert, J. Phys. Chem. B 113, 12565 (2009).

${ }^{10}$ Z. Liu, H. Yan, K. Wang, T. Kuang, J. Zhang, L. Gui, X. An, and W. Chang, Nature 428, 287 (2004).

${ }^{11}$ R. Croce and H. van Amerongen, Nature Chem. Biol. 10, 492 (2014).

${ }^{12}$ E. Songaila, R. Augulis, A. Gelzinis, V. Butkus, A. Gall, C. Büchel, B. Robert, D. Zigmantas, D. Abramavicius, and L. Valkunas, J. Phys. Chem. Lett. 4, 3590 (2013).

${ }^{13}$ T. Brixner, J. Stenger, H. M. Vaswani, M. Cho, R. E. Blankenship, and G. R. Fleming, Nature 434, 625 (2005)

${ }^{14}$ G. S. Schlau-Cohen, T. R. Calhoun, N. S. Ginsberg, E. L. Read, M. Ballottari, R. Bassi, R. van Grondelle, and G. R. Fleming, J. Phys. Chem. B 113, 15352 (2009).

${ }^{15}$ A. Gelzinis, L. Valkunas, F. D. Fuller, J. P. Ogilvie, S. Mukamel, and D. Abramavicius, New J. Phys. 15, 075013 (2013).

${ }^{16}$ F. D. Fuller, J. Pan, A. Gelzinis, V. Butkus, S. S. Senlik, D. E. Wilcox, C. F. Yocum, L. Valkunas, D. Abramavicius, and J. P. Ogilvie, Nature Chem. 6, 706 (2014).

${ }^{17}$ E. Romero, R. Augulis, V. I. Novoderezhkin, M. Ferretti, J. Thieme, D. Zigmantas, and R. van Grondelle, Nature Phys. 10, 676 (2014).

${ }^{18}$ H. Lee, Y.-C. Cheng, and G. Fleming, Science 316, 1462 (2007).

${ }^{19}$ D. Zigmantas, E. L. Read, T. Mančal, T. Brixner, A. T. Gardiner, R. J. Cogdell, and G. R. Fleming, Proc. Natl. Acad. Sci. USA 103, 12672 (2006).

${ }^{20}$ J. Dostál, T. Mančal, R. Augulis, F. Vácha, J. Pšenčik, and D. Zigmantas, J. Am. Chem. Soc. 134, 11611 (2012).

${ }^{21}$ J. M. Anna, E. E. Ostroumov, K. Maghlaoui, J. Barber, and G. D. Scholes, J. Phys. Chem. Lett. 3, 3677 (2012).

${ }^{22}$ E. Collini and G. D. Scholes, Science 323, 369 (2009).

${ }^{23}$ F. Milota, J. Sperling, A. Nemeth, and H. Kauffmann, Chem. Phys. 357, 45 (2009).

${ }^{24}$ D. B. Turner, Y. Hassan, and G. D. Scholes, Nano Lett. 12, 880 (2012).

${ }^{25}$ A. Gelzinis, V. Butkus, E. Songaila, R. Augulis, A. Gall, C. Büchel, B. Robert, D. Abramavicius, D. Zigmantas, and L. Valkunas, Biochim. Biophys. Acta 1847, 241 (2015).

${ }^{26}$ C. Büchel, Biochemistry 42, 13027 (2003).

${ }^{27}$ T. Brixner, I. V. Stiopkin, and G. R. Fleming, Opt. Lett. 29 884 (2004).

${ }^{28}$ R. Augulis and D. Zigmantas, Opt. Express 19, 13126 (2011).

${ }^{29}$ J. D. Hybl, A. W. Albrecht, S. M. G. Faeder, and D. M. Jonas, Chem. Phys. Lett. 297, 307 (1998).

${ }^{30}$ D. M. Jonas, Annu. Rev. Phys. Chem. 54, 425 (2003).

${ }^{31}$ J. K. Gillie and G. J. Small, J. Phys. Chem. 93, 1620 (1989).

${ }^{32}$ V. Butkus, A. Gelzinis, and L. Valkunas, J. Phys. Chem. A 115, 3876 (2011).

${ }^{33}$ D. Abramavicius, Europhys. Lett. 101, 57007 (2013).

${ }^{34}$ J. Han, H. Zhang, and D. Abramavicius, J. Chem. Phys. 139 , 034313 (2013).

${ }^{35}$ J. Du, T. Teramoto, K. Nakata, E. Tokunaga, and T. Kobayashi, Biophys. J. 101, 995 (2011).
${ }^{36}$ G. Panitchayangkoon, D. Voronine, D. Abramavicius, J. Caram, N. Lewis, S. Mukamel, and G. Engel, Proc. Natl. Acad. Sci. USA 108, 20908 (2011).

${ }^{37}$ H. Li, A. D. Bristow, M. E. Siemens, G. Moody, and S. T. Cundiff, Nature Commun. 4, 1390 (2013).

${ }^{38}$ V. Butkus, D. Zigmantas, L. Valkunas, and D. Abramavicius, Chem. Phys. Lett. 545, 40 (2012).

${ }^{39}$ J. Seibt and T. Pullerits, J. Phys. Chem. C 117, 18728 (2013).

${ }^{40}$ M. Ferretti, V. I. Novoderezhkin, E. Romero, R. Augulis, A. Pandit, D. Zigmantas, and R. Grondelle, Phys. Chem. Chem. Phys. 16, 9930 (2014).

${ }^{41}$ F. V. A. Camargo, H. L. Anderson, S. R. Meech, and I. A. Heisler, J. Phys. Chem. A, Article ASAP (2015).

${ }^{42}$ D. Abramavicius, V. Butkus, J. Bujokas, and L. Valkunas, Chem. Phys. 372, 22 (2010).

${ }^{43}$ D. Abramavicius, L. Valkunas, and S. Mukamel, Europhys. Lett. 80, 17005 (2007)

${ }^{44} \mathrm{P}$. Hamm and M. Zanni, Concepts and methods of 2D infrared spectroscopy (Cambridge University Press, 2011).

${ }^{45}$ V. Butkus, J. Alster, E. Bašinskaitè, R. Augulis, P. Neuhaus, H. L. Anderson, L. Valkunas, D. Abramavicius, and Z. D., in preparation.

${ }^{46}$ V. I. Novoderezhkin, E. G. Andrizhiyevskaya, J. P. Dekker, and R. van Grondelle, Biophys. J. 89, 1464 (2005).

${ }^{47}$ V. Butkus, D. Zigmantas, D. Abramavicius, and L. Valkunas, Chem. Phys. Lett. 587, 93 (2013)

${ }^{48}$ V. Butkus, L. Valkunas, and D. Abramavicius, J. Chem. Phys. 140, 034306 (2014).

${ }^{49}$ A. Telfer, A. A. Pascal, L. Bordes, J. Barber, and B. Robert, J. Phys. Chem. B 114, 2255 (2010).

${ }^{50}$ A. Chenu, N. Christensson, H. F. Kauffmann, and T. Mančal, Sci. Rep. 3, 2029 (2013).

${ }^{51}$ O. Rancova and D. Abramavicius, Journal of Physical Chemistry B 118, 7533 (2014)

${ }^{52}$ H. Dong and G. R. Fleming, J. Phys. Chem. B 118, 8956 (2014).

${ }^{53}$ D. Kosumi, M. Kita, R. Fujii, M. Sugisaki, N. Oka, Y. Takaesu, T. Taira, M. Iha, and H. Hashimoto, J. Phys. Chem. Lett. 3, 2659 (2012)

${ }^{54}$ C. D. P. Duffy, J. Chmeliov, M. Macernis, J. Sulskus, L. Valkunas, and A. V. Ruban, J. Phys. Chem. B 117, 10974 (2013).

${ }^{55}$ F. Caruso, A. W. Chin, A. Datta, S. F. Huelga, and M. B. Plenio, J. Chem. Phys. 131, 105106 (2009).

${ }^{56}$ J. Womick and A. Moran, J. Phys. Chem. B 115, 1347 (2011).

${ }^{57}$ A. Kolli, E. O'Reilly, G. Scholes, and A. Olaya-Castro, J. Chem. Phys. 137, 174109 (2012).

${ }^{58}$ C. C. Gradinaru, A. A. Pascal, F. van Mourik, B. Robert, P. Horton, R. van Grondelle, and H. van Amerongen, Biochemistry 37, 1143 (1998).

${ }^{59}$ X. Pan, M. Li, T. Wan, L. Wang, C. Jia, Z. Hou, X. Zhao, J. Zhang, and W. Chang, Nature Struct. Mol. Biol. 18, 309 (2011).

${ }^{60}$ S. Akimoto, A. Teshigahara, M. Yokono, M. Mimuro, R. Nagao, and T. Tomo, Biochim. Biophys. Acta 1837, 1514 (2014). 